\title{
No impact of Asian ethnicity on EORTC QLQ-C30 scores: Group differences and differential item functioning in paroxysmal nocturnal hemoglobinuria
}

\author{
Carolyn E. Schwartz ${ }^{1,2^{*}}$, Roland B. Stark ${ }^{1}$, Katrina Borowiec ${ }^{1,3}$ and Karl-Johan Myren ${ }^{4}$
}

\begin{abstract}
Background: Paroxysmal nocturnal hemoglobinuria (PNH) is a rare, life-threatening terminal-complement-mediated disease resulting in intravascular hemolysis and thrombosis with significant morbidity and premature mortality. There exists no disease-specific quality-of-life (QOL) measure for PNH. Its QOL effects resemble those of hematologic cancers, which supports the use of cancer-specific QOL measures in PNH clinical trials. The European Organisation for Research and Treatment of Cancer (EORTC) QLQ-C30 has published norms for many European and North American countries, but not for Asian countries. We investigated differences by Asian ethnicity in scores and item function on the EORTC QLQ-C30.
\end{abstract}

Methods: This secondary analysis focused on two non-inferiority PNH trials (301 and 302) comparing eculizumab and ravulizumab $(n=441)$. Analysis of covariance examined the main effect of Asian ethnicity on baseline EORTC QLQ-C30 scores, after adjusting for propensity scores encompassing trial, demographic and clinical factors. Mixed modeling of longitudinal data compared subscale scores in Asian vs. non-Asian patients, after propensity adjustment. Differential item function (DIF) was examined using ordinal regression models at baseline and longitudinally, to predict item score from total score, ethnicity, and their interaction to test for uniform DIF (significant main effect for Asian) and non-uniform DIF (significant Asian-by-total-score interaction).

Results: Of the 15 baseline domains, Asian patients scored slightly better on role and emotional functioning and slightly worse on constipation and diarrhea $(0.22<$ Cohen's $d<0.36)$. In longitudinal models, Asians reported slightly higher appetite loss, diarrhea, and financial difficulties than non-Asians $\left(R^{2}\right.$ increment $\left.<0.0005\right)$. There was negligible uniform and non-uniform DIF, i.e., $R^{2} 0$ to 0.018 , far below Zumbo's (1999) criterion of 0.13 . On average there were larger differences from norms for Asians (mean $=0.05, \mathrm{sd}=0.44$ ) than non-Asians (mean $=-0.07, \mathrm{sd}=0.36$ ), but the size and direction of the differences varied considerably by domain, age, and gender.

Conclusion: When compared to norms, Asian patients showed no systematic biases. DIF results supported this finding. We conclude that Asian ethnicity does not impact interpretation of EORTC QLQ-C30 scores.

Keywords: Quality of life, Paroxysmal nocturnal hemoglobinuria, Ethnicity, EORTC, Norms

*Correspondence: carolyn.schwartz@deltaquest.org

1 DeltaQuest Foundation, Inc, 31 Mitchell Road, Concord, MA 01742, USA

Full list of author information is available at the end of the article

\section{Introduction}

The use of patient-reported outcomes (PRO) has amplified the patient's voice in clinical research [1]. While objective indices of health can be very relevant for original author(s) and the source, provide a link to the Creative Commons licence, and indicate if changes were made. The images or other third party material in this article are included in the article's Creative Commons licence, unless indicated otherwise in a credit line to the material. If material is not included in the article's Creative Commons licence and your intended use is not permitted by statutory regulation or exceeds the permitted use, you will need to obtain permission directly from the copyright holder. To view a copy of this licence, visit http://creativecommons.org/licenses/by/4.0/. The Creative Commons Public Domain Dedication waiver (http://creativeco mmons.org/publicdomain/zero/1.0/) applies to the data made available in this article, unless otherwise stated in a credit line to the data. 
assessing the impact of new treatments, they do not tell the whole story. Only the person experiencing the illness and the treatment can evaluate their impact on day-today functioning and internal symptom experience [2]. With the growth in the field of quality-of-life (QOL) research over the past three decades, there are often many options for measuring PROs for relatively common conditions. For rare conditions, however, the choice is more limited. While it is possible to craft measures using items from selected domains developed by the prominent Patient-Reported Outcome Measurement and Information System (PROMIS) [3] and the NeuroQOL systems [4], these systems may lack the language translations needed for international clinical trials, and translation requirements can be arduous and demanding. Developing and validating wholly new measures for rare conditions can be time-consuming and expensive, particularly because the validation process is iterative and federal regulatory agencies have high bars for accepting a PRO's validity for a particular context [5].

It is thus not uncommon for clinical researchers focusing on rare conditions to use well-known and well-characterized legacy measures. With the benefit of time and seniority, legacy measures can be advantageous. They often have normative values across age, gender, region, and diagnosis, which facilitate interpretation within a study and comparison across studies. They may, however, lack normative values for a particular population. The present work focuses on the implementation of a widelyused cancer measure with a non-cancer rare disease.

Paroxysmal nocturnal hemoglobinuria $(\mathrm{PNH})$ is a rare, life-threatening disease resulting in intravascular hemolysis and thrombosis with significant morbidity and premature mortality. With a prevalence of $12-13$ out of one million people [6], $\mathrm{PNH}$ is characterized by uncontrolled activation of the terminal complement pathway leading to intravascular hemolysis and thrombosis [7-10], and it adversely affects QOL with significant morbidity and premature mortality if untreated [11-18]. People with PNH may experience a range of signs or symptoms across bodily systems, including hemoglobinuria, thrombosis, reduced kidney function, abdominal pain, pulmonary hypertension, chest pain, dyspnea, erectile dysfunction in males, end organ damage, and severe fatigue $[12,13$, 19-22].

Clinical trials testing new treatment options for this condition rely on recruitment across many continents and countries. Such trials have relied on well-known legacy cancer measures, including the European Organisation for Research and Treatment of Cancer (EORTC) QLQ-C30 [23]. While the EORTC has available normative information for many European and North American countries, none exist for Asian countries [24, 25].
Normative data for Asian people are not currently available. The PNH clinical trials evaluating ravulizumab contained over 400 people, of whom about $50 \%$ were of Asian ethnicity $[23,26]$. Thus, in order to compare treatments across participants, it would be important to know, first, whether Asian and non-Asian patients have similar scale/subscale mean scores.

In addition to investigating comparable expected values (i.e., mean scores), one would want to examine whether there is evidence of measurement non-invariance or differential item functioning (DIF) [27]. This important step would assess whether people in different ethnic groups respond differently to specific items by being more or less likely to endorse a given item in light of their scale/subscale score. These underlying differences in item response could confound interpretation. If DIF were found, then one could create ethnic-group-specific scoring that adjusts for such measurement non-invariance.

The present work thus aimed to compare Asian and non-Asian people with $\mathrm{PNH}$ on mean scores on the EORTC function and symptom scales and to investigate DIF by ethnicity in the PNH samples. Our null hypothesis would be that there are no differences in mean scores or item response by Asian ethnicity.

\section{Methods \\ Sample}

This secondary analysis utilized data from two $\mathrm{PNH}$ clinical trials $(n=441)$. Both clinical trials were phase-3, open-label studies evaluating the non-inferiority of ravulizumab compared to eculizumab. Trial 301 (ALXN1210-PNH-301) was conducted in PNH patients naïve to complement inhibitors [23]. Trial 302 (ALXN1210-PNH-302) was conducted in PNH patients who were stable on eculizumab for at least six months and of whom half were randomized to switch to ravulizumab and the other half remained on eculizumab [26].

Data available for analysis included longitudinal follow-up from baseline through the randomized 26-week period and also the extension period from 27 to 52 weeks. During the extension period all participants received ravulizumab. (For complete details on trial inclusion and exclusion criteria and procedures see references $[23,26]$.$) The trials were conducted in$ accordance with the provision of the Declaration of Helsinki, the International Conference on Harmonization guidelines for Good Clinical Practice, and applicable regulatory requirements. The trials were approved by the institutional review board at each participating institution. All the patients provided written informed consent before participation. 


\section{Measures}

The EORTC QLQ-C30 is a comprehensive cancer-specific measure that consists of 30 items covering five function subscales (physical, role, emotional, cognitive, and social); nine symptom subscales/items (fatigue, nausea/ vomiting, pain, dyspnea, insomnia, appetite loss, constipation, diarrhea, and financial difficulties); and a global health status/QOL subscale [28, 29]. Higher scores on the function and global health status/QOL scales and lower scores on the symptom scales reflect better health/ QOL. Of note, each individual item's response options, except those for global health status/QOL, moved toward worsening health. In other words, all items other than global health status/QOL were worded such that higher endorsement reflected worsening health. This is specifically relevant for interpreting DIF analyses. Further details of the measure are provided elsewhere [24].

Demographic characteristics collected in the trial datasets were age, years since diagnosis, baseline body mass index, sex, and region. Clinical data included trial, lactate dehydrogenase $(\mathrm{LDH})$ stratum (stratum $1:<1.5 \times$ upper limit of normal (ULN); stratum 2: 1.5-3 $\times$ ULN; stratum 3: $>3 \times U L N)$; packed red blood cells transfusion history (pRBC) stratum (stratum 1: 0 unit pRBC; stratum 2: 1-14 units pRBC; stratum $3:>14$ units $\mathrm{pRBC}$ ); and binary "flags" (indicators) showing presence/absence of immunosuppressant treatment, aplastic anemia, myelodysplastic syndrome, and bone marrow disorder $[23,26]$.

\section{Statistical analysis Sample comparisons}

T-tests and chi-square statistics were used to compare Asian and non-Asian study participants across the demographic and clinical variables. In addition to p-values, effect sizes (ES) were summarized using indicators of variance explained. Cohen's criteria [30] for small, medium, and large ES were used to facilitate interpretation of differences $(>0.01,>0.06$, and $>0.14$ variance explained, respectively).

Propensity Scores. In order to make the soundest comparisons between Asian and non-Asian groups of people with $\mathrm{PNH}$, we created propensity scores, specifically to be used via the covariate-adjustment method [31]. Such scores would be used in subsequent multivariate models to adjust for clinical and demographic variables that might confound the variable of interest, Asian ethnicity. Logistic models predicted Asian vs. non-Asian ethnicity group (dependent variable) with the following variables: age, baseline body mass index, treatment infusion start date, sex, observed LDH stratum, observed pRBC stratum, immune-suppressant treatment flag, aplastic anemia flag, myelodysplastic syndrome flag, bone marrow disorder flag, and trial. Trial was included as a covariate in the propensity score model because patients entering trial 302 had been stable on eculizumab for at least six months and had well-controlled hemolysis prior to randomization. These study patients thus would have better QOL scores at baseline, which may confound subsequent analyses. Further, there were trial differences in proportion of participants who were Asian: $75 \%$ of the Asian patients in the sample were from Trial 301, and 25\% from Trial 302 (Table 1).

\section{Missing-data imputation for propensity scores.}

The type of propensity-score computation we chose was done with logistic rather than mixed models; accordingly, the models used listwise deletion. Consequently, many rows with incomplete sets of covariates would be left out of the propensity-score computation. We thus performed the computation in two stages. In the first stage, we used all 11 covariates and were able to include $95 \%$ of patients. In the second stage, we filled in any missing propensity scores by employing only those 10 covariates that were completely filled in or for which a blank could be treated as its own category rather than a missing. The end result was propensity scores for all 441 patients in the analytic dataset.

\section{Multivariate models}

Analysis of covariance (ANCOVA) [32] was used to evaluate the association of Asian ethnicity with baseline EORTC function and symptom scores, after adjusting for propensity scores. Mixed models [33] were used to evaluate the association of Asian ethnicity with longitudinal EORTC function and symptom scores, after adjusting for propensity scores. Cohen's $d$ [30] was used to facilitate interpretation of differences (small ES $=0.20-0.49$; medium $\mathrm{ES}=0.50-0.79$; large $\mathrm{ES} \geq 0.80$ ) for ANCOVA models, and pseudo- $\mathrm{R}^{2}$ increment to variance explained was used for mixed models (i.e., small $E S=0.010-0.059$; medium $\mathrm{ES}=0.060-0.139$; large $\mathrm{ES} \geq 0.140$ ).

\section{DIF comparisons}

DIF analyses [27, 34] were conducted on the 24 EORTC QLQ-C30 items belonging to scales with at least two items. In theory, when two groups have the same amount of a trait (e.g., cognitive functioning), the likelihood of endorsing an item designed to measure that trait should not differ across groups. This analytic approach tests the hypothesis that people in one group systematically endorse items differently than those in another group, adjusting for the individuals' total score on the scale in question.

The DIF analyses used ordinal logistic regression and involved building three nested models: 
Table 1 Demographics of PNH patients at baseline

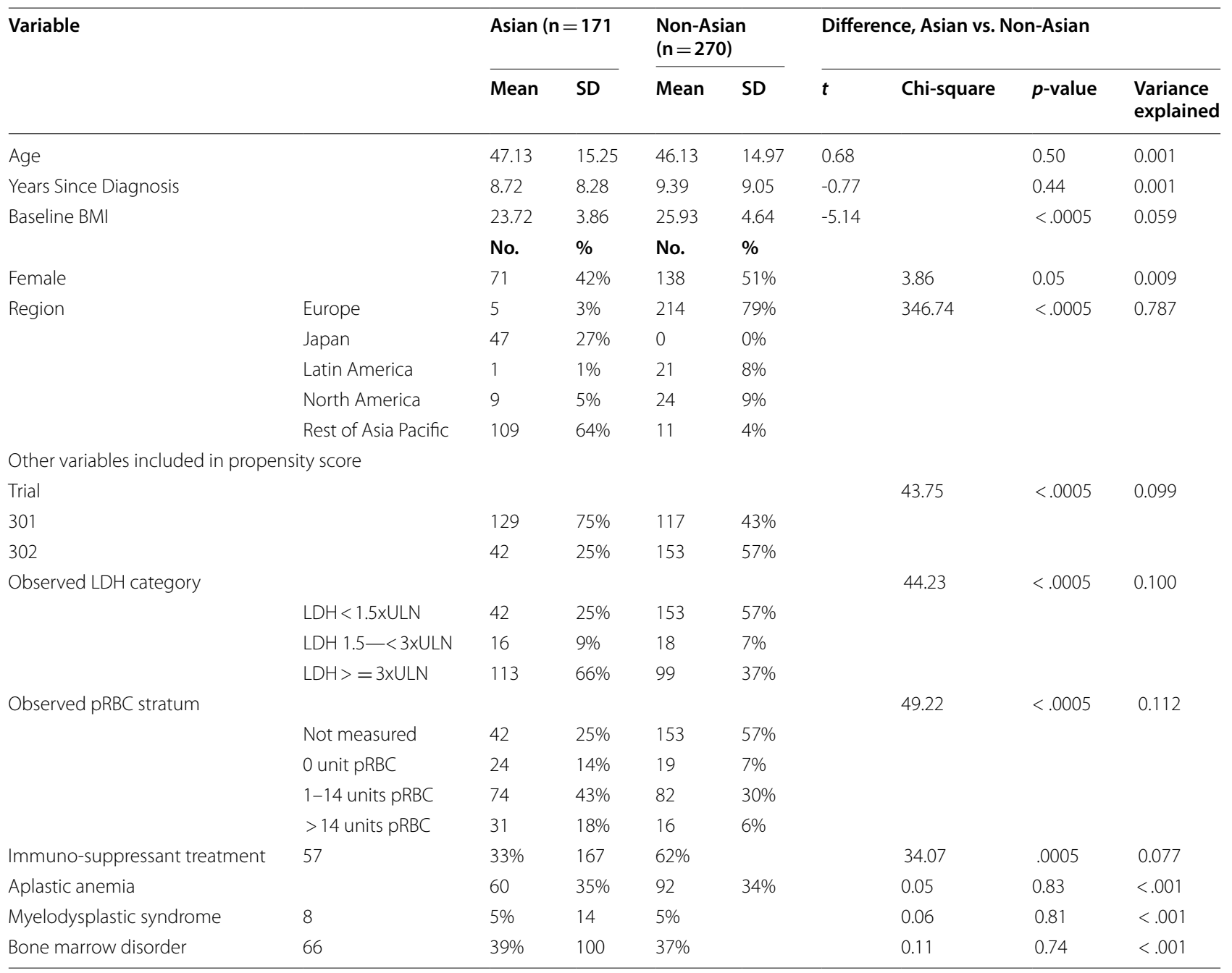

Model 1: $\operatorname{Logit}[P(Y \leq j)]=\alpha_{j}+b_{1}($ Total Score $)$; Model 2: $\operatorname{Logit}[\mathrm{P}(Y \leq j)]=\alpha_{j}+b_{1}$ (Total Score $)+$ $b_{2}$ (Group); and

Model 3: $\operatorname{Logit}[P(Y \leq j)]=\alpha_{j}+b_{1}($ Total Score $)+$ $b_{2}($ Group $)+b_{3}$ (Total Score * Group),

where $\mathrm{P}(\mathrm{Y} \leq \mathrm{j})$ represents the probability that $j$ is the rating-scale response category,

$\alpha_{\mathrm{j}}$ is a regression constant, and each $b$ is a regression coefficient. $^{1}$

The log-likelihood ratio test compares Model 1 versus 2, Model 2 versus 3, and Model 1 versus 3. Uniform DIF

\footnotetext{
${ }^{1}$ Technical note: The standard ordinal logistic regression model parameterization indicates the probability of moving to a lower category for each unit increase in X. Stata transforms the output such that positive coefficients indicate higher probabilities of moving to a "higher" category for each unit increase in $X$, easing interpretation.
}

is characterized by $b_{2}$ being significant and the log likelihood test comparing Models 1 and 2 being significant (i.e., there is a significant main effect for Group). Nonuniform DIF is characterized by $\mathrm{b}_{3}$ being significant and the log-likelihood test comparing Models 2 and 3 being significant (i.e., there is a significant Group-by-totalscore interaction). Uniform and Non-uniform DIF is characterized by the log-likelihood test comparing Models 1 and 3 being significant.

DIF analyses compared scores on baseline data and, separately, with longitudinal data. Uniform DIF analysis results are expressed in terms of a particular group being "favored" on an item, meaning it is "easier" for that group to endorse poor health for that item. The use of the term "easier," as compared to "harder," reflects the centrality of the idea of difficulty in the analysis of item response. Greater item difficulty would mean a higher 
bar for endorsing a particular response option, given one's total score on that domain. We relied on Zumbo's [27] criterion that a pseudo- $R^{2}$ change (i.e., between Models 1 and 2 for uniform DIF; between Models 2 and 3 for non-uniform DIF) of at least $13 \%$ was necessary to indicate substantial DIF. Odds ratios were also obtained to facilitate interpretation. If significant, this type of DIF would reflect that Asians with $\mathrm{PNH}$ were responding differently to the EORTC items as compared to non-Asians with PNH.

SPSS Release 27 [35] and Stata/IC 16.1 [36] were used for all analyses.

\section{Results \\ Sample}

The clinical-trial data used in this secondary analysis included $441 \mathrm{PNH}$ patients of whom 171 were Asian and 270 non-Asian. Table 1 provides descriptive demographic information about the study participants. They were most commonly in their forties, with an average of about nine years since diagnosis, and Asians were similar to non-Asians on these variables. Compared to nonAsian patients in these two trials, there were slightly more females in the Asian patient subgroup. About one quarter of the Asians in the study were from Japan, 64\% were from other parts of Asia or the Pacific (i.e., Australia, Korea, Malaysia, Singapore, Taiwan, Thailand, and Turkey), and just 9\% total were from Europe, North America or Latin America.

Asians were different from non-Asians on a number of clinical variables (Table 1 ). They were more likely to be treatment-naïve (i.e., enrolled in trial 301 rather than 302). Related to this trial inclusion, they were more likely to be in the worst LDH stratum, more likely to have data on transfusion history and be in the higher two pRBC strata, and less likely to be on immuno-suppressive treatment. Asian participants had a lower mean baseline body mass index ( 23.7 vs. 25.9 ). There were no differences by ethnicity on flags for aplastic anemia, myelodysplastic syndrome, or bone marrow disorder.

\section{Propensity scores}

Additional file 1: Table S1 shows parameter coefficients for the propensity score model and provides a Q-Q plot and standardized mean difference of the propensity scores by ethnic group. The calculation was largely driven by $\mathrm{pRBC}$ stratum, treatment infusion start date, sex, and baseline body mass index.

\section{Baseline multivariate models}

Table 2 shows results of the baseline ANCOVA models evaluating the association between Asian ethnicity
Table 2 Results of baseline ANCOVA models with Asian Ethnicity predicting EORTC QLQ-C30 score $(428<=\mathrm{N}<=433)$ *

\begin{tabular}{lcccc}
\hline Subscale & B for Asian group & SE & $\boldsymbol{p}^{* *}$ & Cohen's $\boldsymbol{d}$ \\
\hline $\begin{array}{l}\text { Function scales (higher is better) } \\
\text { GlobalHealthStatus/ }\end{array}$ & -1.11 & & & \\
QOL & & 2.31 & 0.63 & -0.05 \\
Physical functioning & 3.09 & 2.03 & 0.13 & 0.16 \\
Role functioning & 5.86 & 2.91 & $\mathbf{0 . 0 4}$ & 0.22 \\
Emotional functioning & 5.66 & 2.49 & $\mathbf{0 . 0 2}$ & 0.25 \\
Cognitive functioning & -3.05 & 2.45 & 0.22 & -0.14 \\
Social functioning & -1.57 & 2.86 & 0.58 & -0.06 \\
Symptom scales (higher is worse) & -0.48 & & & \\
Fatigue & 0.81 & 2.70 & 0.86 & -0.02 \\
Nausea and vomiting & 0.74 & 1.50 & 0.59 & 0.06 \\
Pain & -1.91 & 2.66 & 0.78 & 0.03 \\
Dyspnoea & 3.10 & 0.54 & -0.07 \\
Insomnia & -1.81 & 3.10 & 0.56 & -0.06 \\
Appetite loss & 1.21 & 2.37 & 0.61 & 0.06 \\
Constipation & 5.21 & 2.24 & $\mathbf{0 . 0 2}$ & 0.25 \\
Diarrhea & 6.56 & 2.01 & $\mathbf{0 . 0 0}$ & 0.36 \\
Financial difficulties & 4.98 & 3.29 & 0.13 & 0.17 \\
\hline
\end{tabular}

${ }^{*}$ Adjusted for propensity score. ${ }^{* *}$ Values $<0.05$ are in bold

and baseline EORTC function and symptom scores, after adjusting for propensity scores. Asians with $\mathrm{PNH}$ reported better role and emotional functioning scores and worse constipation and diarrhea scores than nonAsians. These differences had small ESs.

\section{Longitudinal multivariate models}

Table 3 shows results of the mixed models evaluating the association between Asian ethnicity and EORTC function and symptom scores over time, after adjusting for propensity scores. Asian PNH patients reported worse appetite loss, diarrhea, and financial difficulties than nonAsians, and no differences over time on function scores. These differences did not qualify as small ESs according to Cohen's criteria [30].

\section{Baseline DIF comparisons}

Table 4 shows results of the baseline DIF comparisons. Negligible uniform DIF was detected in six function and three symptom items, but this evidence of DIF was evenly distributed within scales as favoring Asians and nonAsians, effectively cancelling out the effects. One physical function item showed evidence of negligible non-uniform DIF. Six items showed evidence of negligible uniform and non-uniform DIF. In all cases, the magnitude of the change in pseudo- $R^{2}$ accounted for by the group 
Table 3 Results of mixed models with Asian Ethnicity predicting EORTC QLQ-C30 score*

\begin{tabular}{|c|c|c|c|c|}
\hline Subscale & b & SE & $p^{* *}$ & $\begin{array}{l}\text { Pseudo- } R^{2} \\
\text { increment }\end{array}$ \\
\hline \multicolumn{5}{|c|}{ Function scales (higher is better) } \\
\hline GlobalHealthStatus/QOL & -1.29 & 1.71 & 0.45 & -0.0001 \\
\hline Physical functioning & 1.07 & 1.51 & 0.48 & 0.0000 \\
\hline Role functioning & 2.80 & 1.90 & 0.14 & -0.0001 \\
\hline Emotional functioning & 2.55 & 1.78 & 0.15 & 0.0000 \\
\hline Cognitive functioning & -2.25 & 1.81 & 0.21 & 0.0001 \\
\hline Social functioning & -1.80 & 1.95 & 0.36 & 0.0001 \\
\hline \multicolumn{5}{|c|}{ Symptom scales (higher is worse) } \\
\hline Fatigue & 0.45 & 1.94 & 0.82 & 0.0000 \\
\hline Nausea and vomiting & 0.61 & 0.61 & 0.32 & -0.0001 \\
\hline Pain & 0.63 & 1.49 & 0.67 & 0.0000 \\
\hline Dyspnoea & -2.87 & 2.02 & 0.15 & -0.0002 \\
\hline Insomnia & -1.67 & 2.13 & 0.43 & -0.0002 \\
\hline Appetite loss & 2.98 & 1.27 & 0.02 & -0.0005 \\
\hline Constipation & 3.06 & 1.61 & 0.06 & 0.0004 \\
\hline Diarrhea & 3.00 & 1.25 & 0.02 & 0.0003 \\
\hline Financial difficulties & 4.93 & 2.32 & 0.03 & 0.0002 \\
\hline
\end{tabular}

${ }^{*}$ Adjusted for propensity score. ${ }^{* *}$ Values $<0.05$ are in bold

effect and the group-by-total score interaction effect (i.e., variables used to identify DIF) was substantially smaller than Zumbo's criterion of $13 \%$. Thus, no notable DIF by ethnicity was detected in the baseline comparisons.

\section{Longitudinal DIF comparisons}

Table 5 shows results of the longitudinal comparisons. Uniform DIF was detected in six function and two symptom items, but again this evidence of DIF was evenly distributed within scales as favoring Asians and non-Asians, effectively cancelling out the effects. Non-uniform DIF was detected in three of the four emotional function items. For "tense" and "irritable", Asians were less likely to endorse poor health at moderate emotional health. In contrast, for "depressed", Asians were more likely to endorse poor health at low and moderate levels of emotional health. In other words, Asians with $\mathrm{PNH}$ who had a total score indicative of moderate Emotional Functioning were less likely to endorse being tense or irritable and more likely to endorse being depressed. These different non-uniform DIF directions would have a negligible impact.

\section{Discussion}

To our knowledge, this is the first study to address Asian ethnicity and item response on the EORTC. The present study found small ES differences in EORTC scale scores between Asians and non-Asians at baseline and over time, suggestive of slightly better role and emotional functioning at baseline and slightly worse appetite loss, diarrhea, and financial difficulties over time. These analyses adjusted for differences in demographic and clinical risk factors, and they revealed at most small differences at baseline and negligible differences over time. Small effect sizes do not generally meet the threshold for "clinical significance" [37]. Further, only negligible DIF effects were detected at baseline or over time, and these effects did not systematically "favor" one ethnic group over the other. Thus, while there were some ethnicity-related differences, they do not appear to reflect a systematic bias in EORTC scores or item response.

These results would suggest that interpreting differences in Asian versus non-Asian people with $\mathrm{PNH}$ is not confounded with differences in item functioning across groups. In general, after accounting for differences in total functional problems and symptom burdens, respectively, no one ethnic group was more likely to endorse specific functional problems or symptom burdens. Accordingly, digestive symptoms at baseline seem more problematic to Asians, and for them over time such symptoms and financial difficulties are, negligibly, more problematic. Such digestive-symptom differences may be worthy of targeted intervention for symptom relief. In contrast, the finding that Asians reported better role and emotional functioning at baseline was not true over the full course of follow-up, so it may not have much clinical importance.

While the study has the advantage of a relatively large sample of this rare condition across diverse geographic regions, the limitations must be acknowledged. First, there is limited demographic information about trial participants, and so testing hypotheses about other variables that might be responsible for functioning or symptomburden differences is not possible. For example, information on cultural characteristics is not known. The relationship between ethnicity and QOL outcomes could be mediated or moderated by health behaviors (e.g., smoking, exercise, dietary habits), or socioeconomic status (e.g., employment status, education level, marital status). Such limitations are common disadvantages in secondary data analysis. Second, six of the nine scales included only two survey items, and therefore, findings from the DIF analyses should be interpreted with some caution. 


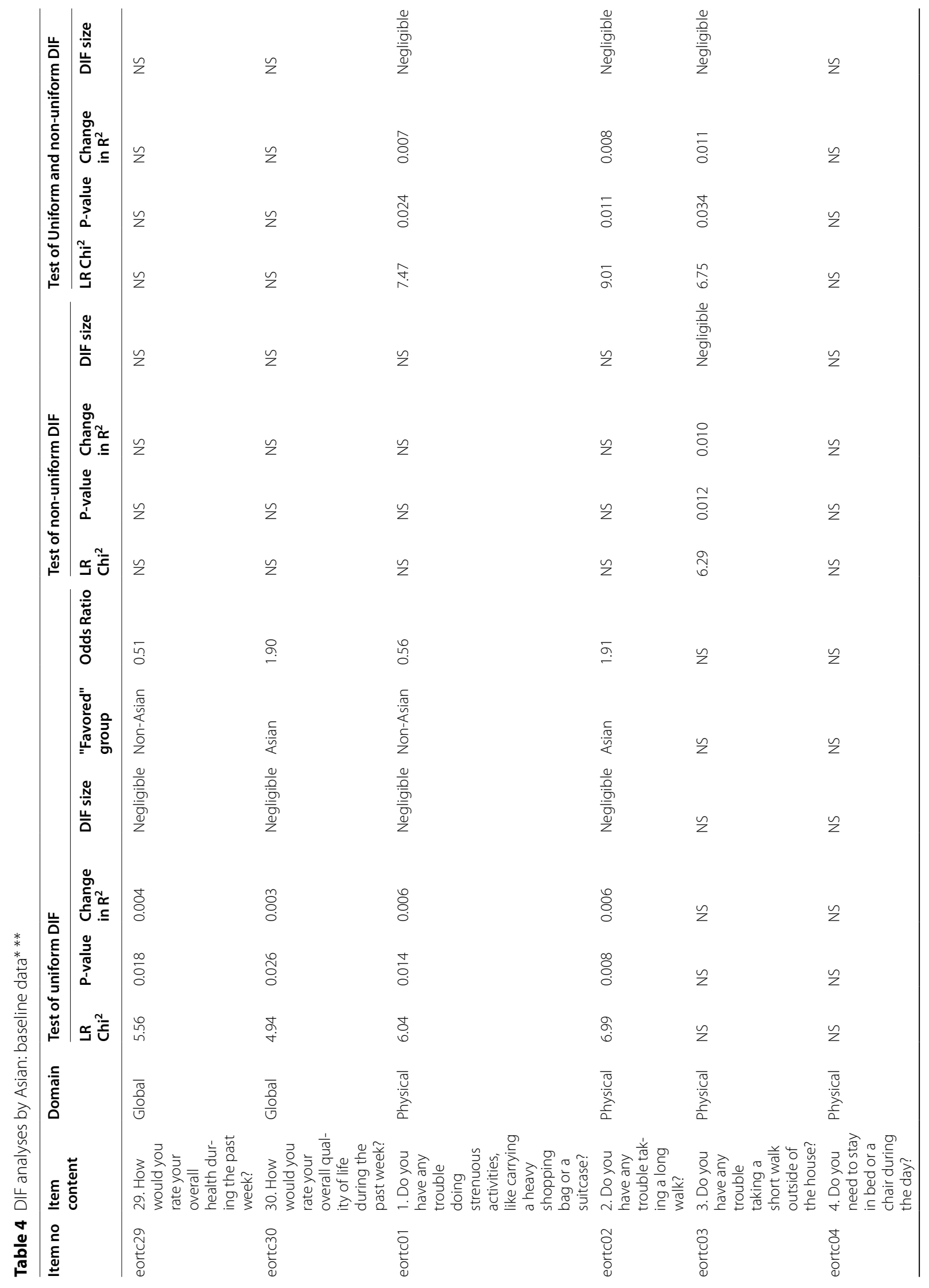




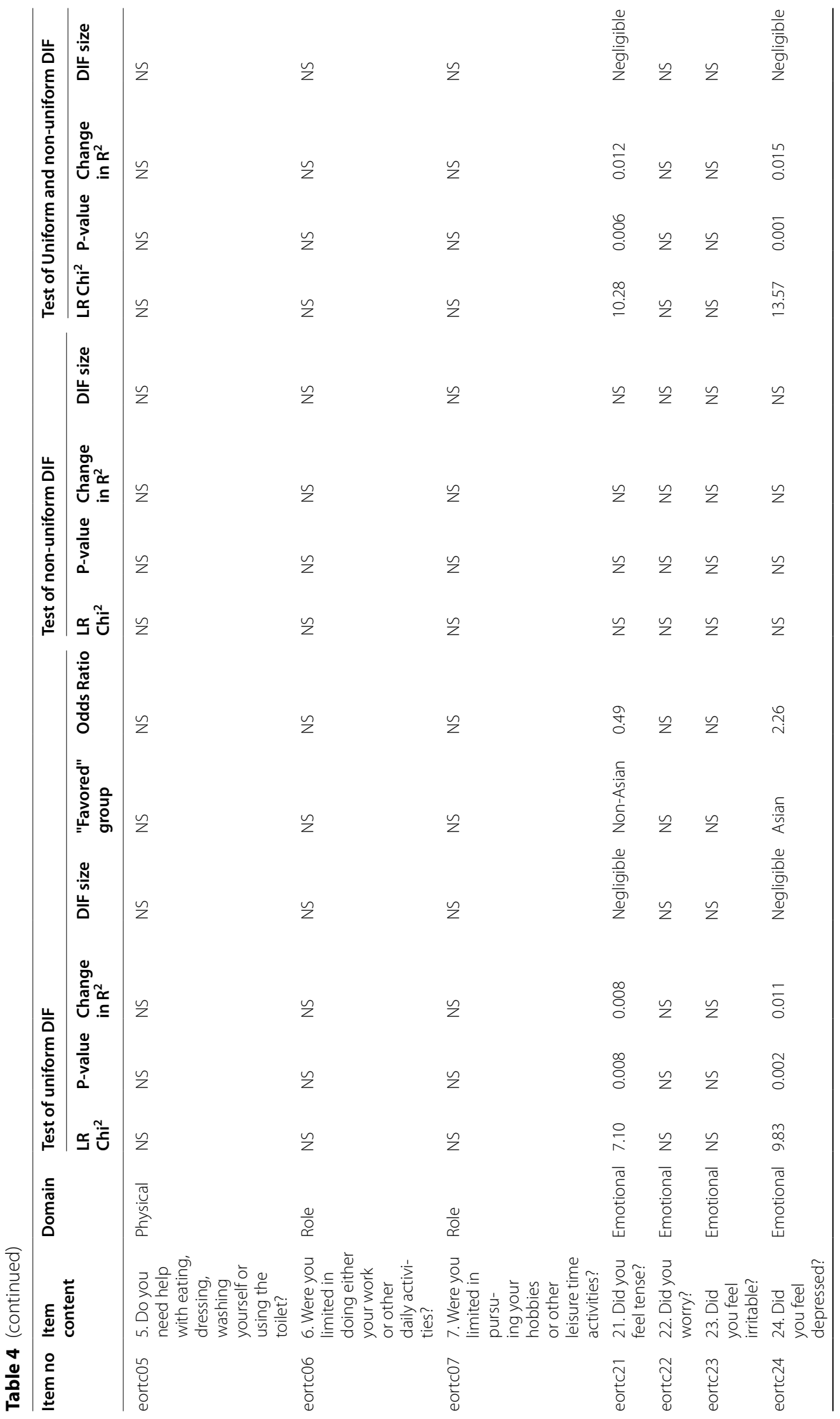




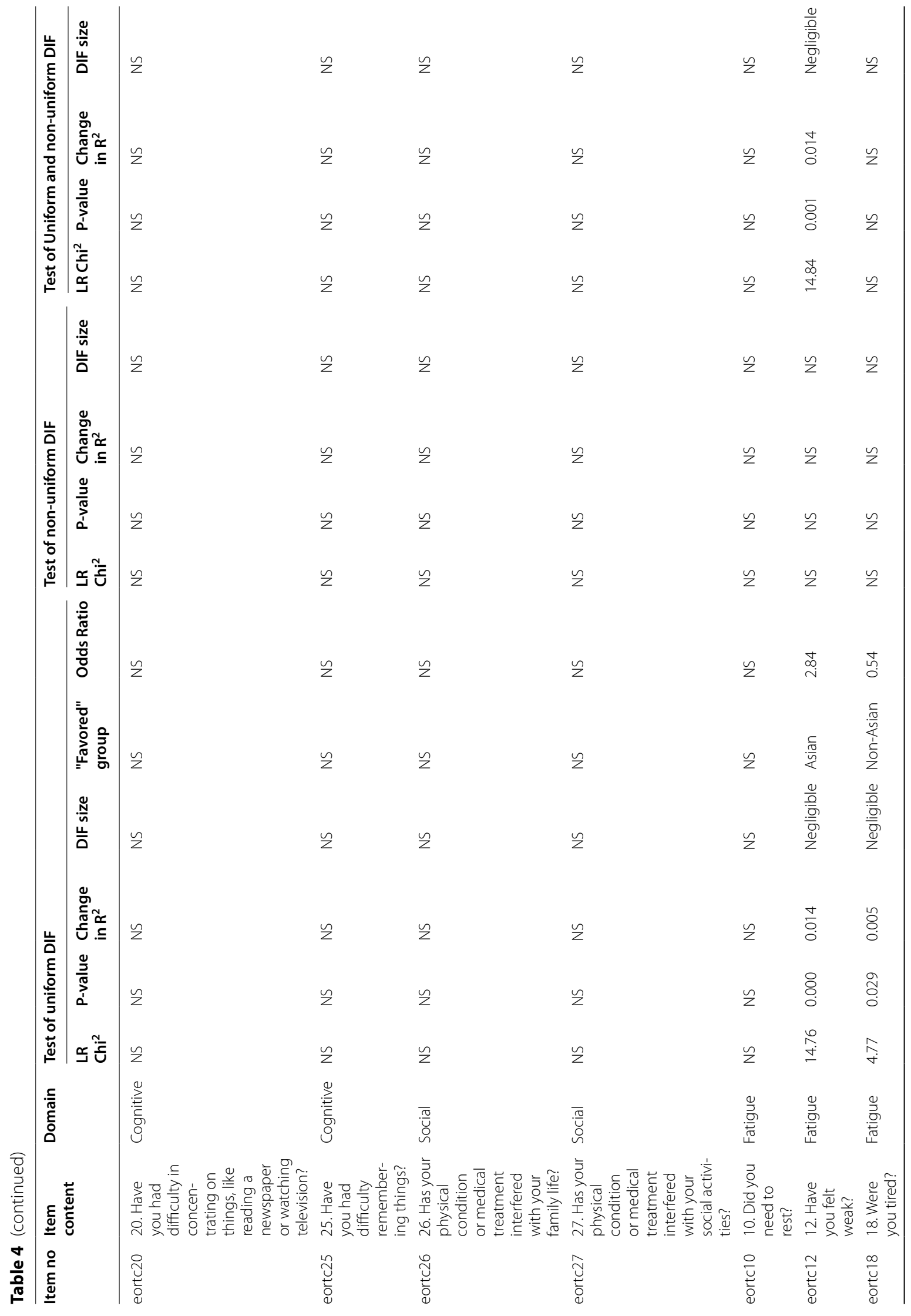




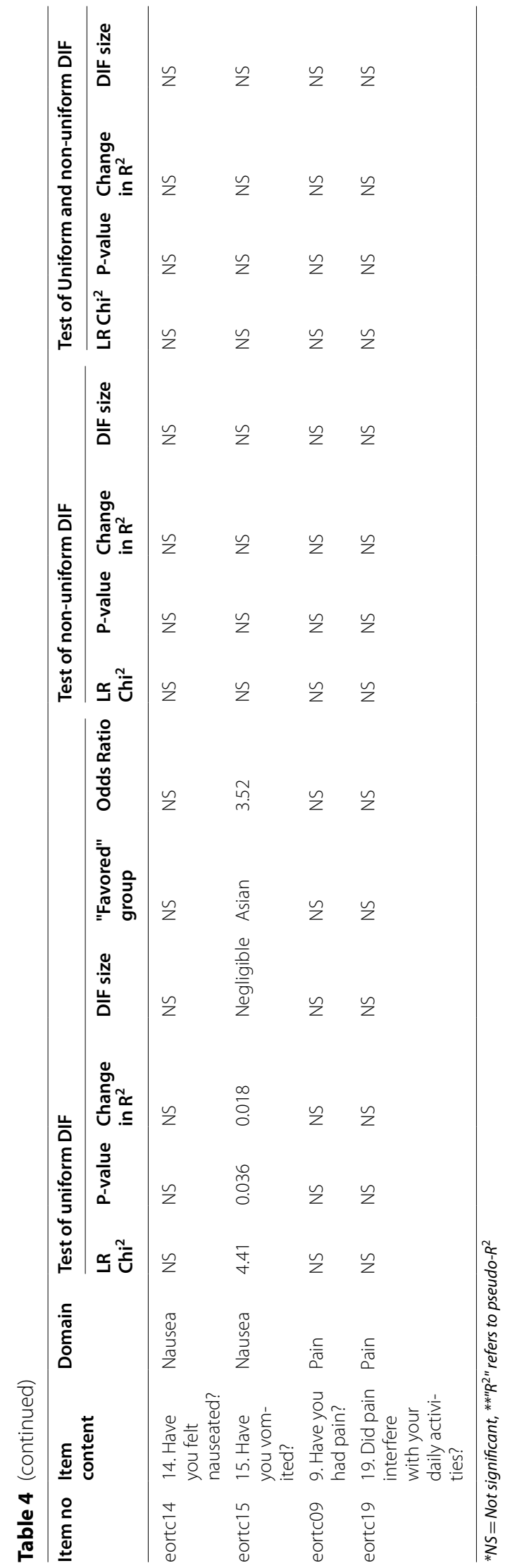


Table 5 DIF Analyses by Asian: longitudinal data

\begin{tabular}{|c|c|c|c|c|c|c|}
\hline \multirow[t]{2}{*}{ Item no } & \multirow[t]{2}{*}{ Item content } & \multirow[t]{2}{*}{ Domain } & \multicolumn{3}{|c|}{ Test of uniform DIF } & \multirow{2}{*}{$\begin{array}{l}\text { Test of non-uniform DIF } \\
\text { P-value (on Interaction term) }\end{array}$} \\
\hline & & & $\begin{array}{l}\text { P-value } \\
\text { (group } \\
\text { effect) }\end{array}$ & "Favored" group & Odds Ratio & \\
\hline eortc29 & $\begin{array}{l}\text { 29. How would you rate your overall health dur- } \\
\text { ing the past week? }\end{array}$ & Global & NS & NS & NS & NS \\
\hline eortc30 & $\begin{array}{l}\text { 30. How would you rate your overall quality of } \\
\text { life during the past week? }\end{array}$ & Global & NS & NS & NS & NS \\
\hline eortc01 & $\begin{array}{l}\text { 1. Do you have any trouble doing strenuous } \\
\text { activities, like carrying a heavy shopping bag or } \\
\text { a suitcase? }\end{array}$ & Physical & $p=.001$ & Non-Asian & 0.43 & NS \\
\hline eortc02 & 2. Do you have any trouble taking a long walk? & Physical & $p=.005$ & Asian & 2.13 & NS \\
\hline eortc03 & $\begin{array}{l}\text { 3. Do you have any trouble taking a short walk } \\
\text { outside of the house? }\end{array}$ & Physical & NS & NS & NS & NS \\
\hline eortc04 & $\begin{array}{l}\text { 4. Do you need to stay in bed or a chair during } \\
\text { the day? }\end{array}$ & Physical & NS & NS & NS & NS \\
\hline eortc05 & $\begin{array}{l}\text { 5. Do you need help with eating, dressing, wash- } \\
\text { ing yourself or using the toilet? }\end{array}$ & Physical & NS & NS & NS & NS \\
\hline eortc06 & $\begin{array}{l}\text { 6. Were you limited in doing either your work or } \\
\text { other daily activities? }\end{array}$ & Role & NS & NS & NS & NS \\
\hline eortc07 & $\begin{array}{l}\text { 7. Were you limited in pursuing your hobbies or } \\
\text { other leisure time activities? }\end{array}$ & Role & NS & NS & NS & NS \\
\hline eortc21 & 21. Did you feel tense? & Emotional & $p=.001$ & Non-Asian & 0.44 & $p=.011$ \\
\hline eortc22 & 22. Did you worry? & Emotional & NS & NS & NS & NS \\
\hline eortc23 & 23. Did you feel irritable? & Emotional & NS & NS & NS & $p=.035$ \\
\hline eortc24 & 24. Did you feel depressed? & Emotional & $p<.001$ & Asian & 3.21 & $\mathrm{p}<.001$ \\
\hline eortc20 & $\begin{array}{l}\text { 20. Have you had difficulty in concentrating on } \\
\text { things, like reading a newspaper or watching } \\
\text { television? }\end{array}$ & Cognitive & $p=.001$ & Non-Asian & 0.36 & NS \\
\hline eortc25 & $\begin{array}{l}\text { 25. Have you had difficulty remembering } \\
\text { things? }\end{array}$ & Cognitive & $p<.001$ & Asian & 3.53 & NS \\
\hline eortc26 & $\begin{array}{l}\text { 26. Has your physical condition or medical treat- } \\
\text { ment interfered with your family life? }\end{array}$ & Social & NS & NS & NS & NS \\
\hline eortc27 & $\begin{array}{l}\text { 27. Has your physical condition or medical treat- } \\
\text { ment interfered with your social activities? }\end{array}$ & Social & NS & NS & NS & NS \\
\hline eortc10 & 10. Did you need to rest? & Fatigue & NS & NS & NS & NS \\
\hline eortc12 & 12. Have you felt weak? & Fatigue & $p=.002$ & Asian & 2.20 & NS \\
\hline eortc18 & 18. Were you tired? & Fatigue & $p=.001$ & Non-Asian & 0.50 & NS \\
\hline eortc14 & 14. Have you felt nauseated? & Nausea & NS & NS & NS & NS \\
\hline eortc15 & 15. Have you vomited? & Nausea & NS & NS & NS & NS \\
\hline eortc09 & 9. Have you had pain? & Pain & NS & NS & NS & NS \\
\hline eortc19 & 19. Did pain interfere with your daily activities? & Pain & NS & NS & NS & NS \\
\hline
\end{tabular}

$N S=$ Not significant

\section{Conclusions}

In summary, we did not find evidence of systematic differences or biases between Asian and non-Asian patients with PNH. We conclude that Asian ethnicity is not likely to impact interpretation of EORTC QLQ-C30 scores.

\section{Abbreviations}

DIF: Differential item functioning; EORTC QLQ-C30: European Organisation for Research and Treatment of Cancer Quality of Life Questionnaire-C30 measure; ANCOVA: Analysis of covariance; MANCOVA: Multivariate analysis of covariance; PNH: Paroxysmal nocturnal hemoglobinuria; PRO: Patient-reported outcome; QOL: Quality of life.

\section{Supplementary Information}

The online version contains supplementary material available at https://doi. org/10.1186/s12955-021-01860-3.

Additional file 1. More Information about the Propensity Score. 


\section{Acknowledgements}

We are grateful for data management assistance from John Kaye, Jimmy Wang, and Ji Yu, and for helpful input from Dr. Francesca Martinelli, Dr. Anita Hill, and Dr. Rodrigo Pavani. The authors thank the European Organization for Research and Treatment of Cancer for permission to use the aggregated data provided in Tables in the Nolte et al. (2019) publication. The contents of this publication and methods used are solely the responsibility of the authors and do not necessarily represent the official views of the EORTC.

\section{Authors' contributions}

CES and RBS designed the research study. CES, RBS, and KB analyzed the data with relevant input from KJM. CES wrote the paper and RBS, KB, and KJM edited the manuscript. All authors read and approved the final manuscript.

\section{Funding}

This work was funded in part by Alexion Pharmaceuticals to Dr. Carolyn E. Schwartz at DeltaQuest Foundation.

\section{Availability of data and material}

Alexion will consider requests for disclosure of clinical study participant-level data provided that participant privacy is assured through methods like data de-identification, pseudonymization, or anonymization (as required by applicable law), and if such disclosure was included in the relevant study informed consent form or similar documentation. Qualified academic investigators may request participant-level clinical data and supporting documents (statistical analysis plan and protocol) pertaining to Alexion-sponsored studies. Further details regarding data availability and instructions for requesting information are available in the Alexion Clinical Trials Disclosure and Transparency Policy at https://alexion.com/our-research/research-and-development.

\section{Code availability}

Requests for software code will be considered, and if they are deemed reasonable code will be made available.

\section{Declarations}

\section{Ethics approval and consent to participate}

The trial was conducted in accordance with the provision of the Declaration of Helsinki, the International Conference on Harmonization guidelines for Good Clinical Practice, and applicable regulatory requirements. The trial was approved by the institutional review board at each participating institution. All the patients provided written informed consent before participation. For the EORTC general-population data, ethical approval was not sought as this study was solely based on panel research data collected by GFK SE. The survey conformed to the required ethical standards by obtaining written informed consent from all participants and collecting data completely anonymously. All participants provided informed consent prior to beginning the survey.

\section{Consent to publish}

All participants provided permission in the informed consent for their aggregated data being used in research publications.

\section{Competing interests}

Dr. Schwartz and Mr. Stark are employees of and Ms. Borowiec an independent contractor of DeltaQuest Foundation, which received research funding for the work reported herein. Mr. Myren is an employee of Alexion Pharmaceuticals which developed the drugs being evaluated.

\section{Author details}

'DeltaQuest Foundation, Inc, 31 Mitchell Road, Concord, MA 01742, USA.

2 Departments of Medicine and Orthopaedic Surgery, Tufts University Medical School, Boston, MA, USA. ${ }^{3}$ Department of Measurement, Evaluation, Statistics, \& Assessment, Boston College Lynch School of Education and Human Development, Chestnut Hill, MA, USA. ${ }^{4}$ Health Economics and Outcome Research, Alexion Pharmaceuticals, Inc, Stockholm, Sweden.

Received: 7 June 2021 Accepted: 13 September 2021

Published online: 28 September 2021

\section{References}

1. LeBlanc TW, Abernethy AP. Patient-reported outcomes in cancer care-hearing the patient voice at greater volume. Nat Rev Clin Oncol. 2017;14(12):763-72.

2. Patrick DL, Burke LB, Powers JH, Scott JA, Rock EP, Dawisha S, et al. Patientreported outcomes to support medical product labeling claims: FDA perspective. Value in Health. 2007;10:S125-37.

3. Cella D, Riley W, Stone A, Rothrock N, Reeve B, Yount S, et al. The PatientReported Outcomes Measurement Information System (PROMIS) developed and tested its first wave of adult self-reported health outcome item banks: 2005-2008. J Clin Epidemiol. 2010;63(11):1179-94.

4. National Institute of Neurological Disorders and Stroke (2010). User Manual for the Quality of Life in Neurological Disorders (Neuro-QOL) Measures, version 1.0

5. Speight, J., \& Barendse, S. M. (2010). FDA guidance on patient reported outcomes. British Medical Journal Publishing Group.

6. Jalbert JJ, Chaudhari U, Zhang H, Weyne J, Shammo JM. Epidemiology of PNH and real-world treatment patterns following an incident PNH diagnosis in the US. Blood. 2019;134:3407. https://doi.org/10.1182/ blood-2019-125867.

7. Brodsky RA. Paroxysmal nocturnal hemoglobinuria. Blood. 2014;124(18):2804-11.

8. Holguin M, Fredrick L, Bernshaw N, Wilcox L, Parker C. Isolation and characterization of a membrane protein from normal human erythrocytes that inhibits reactive lysis of the erythrocytes of paroxysmal nocturnal hemoglobinuria. J Clin Investig. 1989;84(1):7-17.

9. Jang JH, Kim JS, Yoon S-S, Lee J-H, Kim Y-K, Jo D-Y, et al. Predictive factors of mortality in population of patients with paroxysmal nocturnal hemoglobinuria (PNH): results from a Korean PNH registry. J Korean Med Sci. 2016;31(2):214

10. World Health Organization (2010). ICD-10.

11. Schrezenmeier H, Muus P, Socié G, Szer J, Urbano-Ispizua A, Maciejewski $J$, et al. Baseline characteristics and disease burden in patients in the International Paroxysmal Nocturnal Hemoglobinuria Registry. Haematologica. 2014;99(5):922.

12. Hillmen P, Lewis S, Bessler M, Luzzatto L, Dacie JV. Natural history of paroxysmal nocturnal hemoglobinuria. N Engl J Med. 1995;333(19):1253-8.

13. Nishimura J-I, Kanakura Y, Ware RE, Shichishima T, Nakakuma H, Ninomiya $\mathrm{H}$, et al. Clinical course and flow cytometric analysis of paroxysmal nocturnal hemoglobinuria in the United States and Japan. Medicine. 2004;83(3):193-207.

14. Hillmen P, Muus $P$, Röth A, Elebute MO, Risitano AM, Schrezenmeier H, et al. Long-term safety and efficacy of sustained eculizumab treatment in patients with paroxysmal nocturnal haemoglobinuria. Br J Haematol. 2013;162(1):62-73.

15. Loschi M, Porcher R, Barraco F, Terriou L, Mohty M, De Guibert S, et al. Impact of eculizumab treatment on paroxysmal nocturnal hemoglobinuria: a treatment versus no-treatment study. Am J Hematol. 2016;91(4):366-70.

16. Socié G, Mary J-Y, de Gramont A, Rio B, Leporrier M, Rose C, et al. Paroxysmal nocturnal haemoglobinuria: long-term follow-up and prognostic factors. Lancet. 1996;348(9027):573-7.

17. Kelly RJ, Hill A, Arnold LM, Brooksbank GL, Richards SJ, Cullen M, et al. Long-term treatment with eculizumab in paroxysmal nocturnal hemoglobinuria: sustained efficacy and improved survival. Blood. 2011;117(25):6786-92.

18. Yenerel MN, Muus P, Wilson A, Szer J. Clinical course and disease burden in patients with paroxysmal nocturnal hemoglobinuria by hemolytic status. Blood Cells Mol Dis. 2017;65:29-34.

19. Hill A, Rother RP, Wang X, Morris J, Sidney M, Quinn-Senger K, Kelly R, et al. Effect of eculizumab on haemolysis-associated nitric oxide depletion, dyspnoea, and measures of pulmonary hypertension in patients with paroxysmal nocturnal haemoglobinuria. Br J Haematol. 2010;149(3):414-25.

20. Hillmen P, Elebute M, Kelly R, Urbano-Ispizua A, Hill A, Rother RP, et al. Long-term effect of the complement inhibitor eculizumab on kidney function in patients with paroxysmal nocturnal hemoglobinuria. Am J Hematol. 2010;85(8):553-9.

21. Parker C, Omine M, Richards S, Nishimura J-I, Bessler M, Ware R, et al. Diagnosis and management of paroxysmal nocturnal hemoglobinuria. Blood. 2005;106(12):3699-709. 
22. Weitz I, Meyers G, Lamy T, Cahn JY, Uranga M, García Vela J, et al. Cross-sectional validation study of patient-reported outcomes in patients with paroxysmal nocturnal haemoglobinuria. Intern Med J. 2013;43(3):298-307.

23. Lee JW, Sicre de Fontbrune F, Lee WL, L., Pessoa, V., Gualandro, S., Füreder, W., et al. Ravulizumab (ALXN1210) vs eculizumab in adult patients with PNH naive to complement inhibitors: the 301 study. Blood. 2019;133(6):530-9.

24. Liegl G, Petersen M, Groenvold M, Aaronson N, Costantini A, Fayers P, et al. Establishing the European Norm for the health-related quality of life domains of the computer-adaptive test EORTC CAT Core. Eur J Cancer. 2019;107:133-41.

25. Nolte S, Liegl G, Petersen M, Aaronson N, Costantini A, Fayers P, et al. General population normative data for the EORTC QLQ-C30 health-related quality of life questionnaire based on 15,386 persons across 13 European countries, Canada and the Unites States. Eur J Cancer. 2019;107:153-63.

26. Kulasekararaj AG, Hill A, Rottinghaus ST, Langemeijer S, Wells R, Gonzalez-Fernandez FA, et al. Ravulizumab (ALXN1210) vs eculizumab in C5-inhibitor-experienced adult patients with PNH: the 302 study. Blood. 2019;133(6):540-9.

27. Zumbo, B. D. (1999). A Handbook on the Theory and Methods of Differential Item Functioning (DIF): Logistic Regression Modeling as a Unitary Framework for Binary and Likert-Type (Ordinal) Item Scores. Ottawa, ON: Directorate of Human Resources Research and Evaluation, Department of National Defense.

28. Fayers, P., Bottomley, A., Group, E. Q. o. L. Quality of life research within the EORTC — the EORTC QLQ-C30. Eur J Cancer. 2002;38:125-33.
29. Aaronson NK, Ahmedzai S, Bergman B, Bullinger M, Cull A, Duez NJ, et al. The European Organization for Research and Treatment of Cancer QLQC30: a quality-of-life instrument for use in international clinical trials in oncology. J Natl Cancer Inst. 1993;85(5):365-76.

30. Cohen J. A power primer. Psychol Bull. 1992;112:155-9.

31. Austin P. An Introduction to Propensity Score Methods for Reducing the Effects of Confounding in Observational Studies. Multivar Behav Res. 2011;46(3):399-424.

32. Zar, J. H. (1984). Analysis of Covariance. In Biostatistical Analysis (Second Edition ed., pp. 300-302). Englewood Cliffs, NJ: Prentice Hall.

33. Laird NM, Ware JH. Random-effects models for longitudinal data. Biometrics. 1982;38(4):963-74.

34. Swaminathan $\mathrm{H}$, Rogers $\mathrm{HJ}$. Detecting differential item functioning using logistic regression procedures. J Educ Meas. 1990;27(4):361-70.

35. IBM. IBM SPSS Statistics for Windows. 27th ed. Armonk, NY: IBM Corp; 2020.

36. StataCorp (2019). Stata Statistical Software: Release 16.1. College Station, TX: StataCorp LLC.

37. Norman GR, Sloan JA, Wyrwich KW. Interpretation of changes in healthrelated quality of life: the remarkable universality of half a standard deviation. Med Care. 2003;41(5):582-92.

\section{Publisher's Note}

Springer Nature remains neutral with regard to jurisdictional claims in published maps and institutional affiliations.
Ready to submit your research? Choose BMC and benefit from:

- fast, convenient online submission

- thorough peer review by experienced researchers in your field

- rapid publication on acceptance

- support for research data, including large and complex data types

- gold Open Access which fosters wider collaboration and increased citations

- maximum visibility for your research: over 100M website views per year

At BMC, research is always in progress.

Learn more biomedcentral.com/submissions 